\title{
Western blot analysis of cells encapsulated in self-assembling peptide hydrogels
}

\author{
Kyle A. Burgess ${ }^{1,2}$, Aline F. Miller,2,3, Delvac Oceandy ${ }^{4}$, and Alberto Saiani ${ }^{1,2, *}$ \\ ${ }^{1}$ School of Materials, The University of Manchester, Manchester, UK, ${ }^{2}$ Manchester Institute of \\ Biotechnology, The University of Manchester, Manchester, UK, ${ }^{3}$ School of Chemical Engineering and \\ Analytical Sciences, The University of Manchester, Manchester, UK, and ${ }^{4}$ Division of Cardiovascular \\ Sciences, The University of Manchester, Manchester, UK.
}

BioTechniques 63:253-260 (December 2017) doi 10.2144/000114617

Keywords: self-assembling peptide hydrogels, western blot analysis

Supplementary material for this article is available at www.BioTechniques.com/article/114617.

Continuous optimization of in vitro analytical techniques is ever more important, especially given the development of new materials for tissue engineering studies. In particular, isolation of cellular components for downstream applications is often hindered by the presence of biomaterials, presenting a major obstacle in understanding how cell-matrix interactions influence cell behavior. Here, we describe an approach for western blot analysis of cells that have been encapsulated in self-assembling peptide hydrogels (SAPHs), which highlights the need for complete solubilization of the hydrogel construct. We demonstrate that both the choice of buffer and multiple cycles of sonication are vital in obtaining complete solubilization, thereby enabling the detection of proteins otherwise lost to SAP aggregation. Moreover, we show that the presence of self-assembling peptides (SAPs) does not interfere with the standard immunoblotting technique, offering the potential for use in more full-scale proteomic studies.

Over the last 40 years (1), western blot analysis has become a wide-spread technique used for a range of purposes, including identification and quantification of proteins, determination of proteinprotein interactions, and detection of post-translational proteins modifications, to name a few (2). The term "western blot" is used to describe the entire process from protein extraction, solubilization, and size separation through to membrane transfer and subsequent antibody detection (3). As with any technique, the accuracy of the results depends heavily on the sample preparation and, in this case, specifically on the recovery and solubilization of cellular proteins for downstream analysis (4). In general, extracting proteins from cell samples relies on the ability to lyse cells in a buffer that promotes protein solubilization. The choice of buffer depends on sample type (5-7), protein of interest (8), and downstream application. Typical buffer components include detergents (anionic, cationic, and non-ionic), salts, reducing agents, pH-buffering compounds, and protease and phosphatase inhibitors (2). Sample-specific optimization is an intrinsic part of method development. In the past two decades, there has been a move from 2-D to 3-D cell culture methods, with a view to recapitulate the native 3-D cellular niche in vitro. As a result, a variety of new biomaterials have appeared in the literature that allow the 3-D encapsulation of cells (9). Determining how these biomaterials might interfere with different analytical techniques is important in selecting and modifying current protocols to obtain accurate and reliable results.

Recently, hydrogels have become increasingly popular as biomaterials used to encapsulate cells for a range of applications, from 3-D cell culture to in vivo cell delivery $(10,11)$. The term hydrogel describes a well-hydrated, porous scaffold which can be fabricated from an array of natural and/or synthetic polymers. Here, self-assembling peptide hydrogels (SAPHs) are of particular interest. A variety of self-assembling peptide (SAP) designs can be found in the literature; one of the most popular, developed by Zhang's group, is based on a pattern of alternating hydrophilic and hydrophobic amino acids $(12,13)$. These peptides (typically 8-16 amino acids long) assemble in water to form $\beta$-sheet rich fibers that, when above the critical gelation concentration (CGC), entangle and/or associate/aggregate to form a 3-D percolated, nanofibrillar network that traps water (i.e., a hydrogel). Examples of SAPHs exploiting Zhang's

\section{METHOD SUMMARY}

Following multiple cycles of sonication, the use of urea/thiourea-unlike radioimmunoprecipitation assay buffer (RIPA) buffer-completely solubilizes both self-assembling peptide hydrogels (SAPHs) and cellular protein from encapsulated cells. The presence of self-assembling peptides (SAPs) did not interfere with PAGE or subsequent antibody detection, thereby enabling western blot analysis of in situ cells. 
peptide design include: RADA (14), KLD (15), FKE (16,17), and Q11 (18), among many others. These SAPHs provide a flexible platform for cell culture, as the properties (mechanical and functional) can be tailored by simply altering the peptide concentration, formulation, and amino acid sequence $(19,20)$. This family of SAPHs has already been shown to support the culture of many different cell lineages, including osteoblasts (21), chondrocytes $(15,20)$, and endothelial cells (22).

For hydrogels composed of naturally occurring extracellular matrix (ECM) molecules, little modification to current protocols is required, as intact cells can be isolated through enzyme-specific degradation using cell-derived matrix metalloproteinases (MMPs) such as collagenase (collagen) (23), nattokinase (fibrin) (24), and hyaluronidase (hyaluronic acid) (25). However, the degradation of syntheticbased polymers represents more of a challenge, although many synthetic materials are being engineered to contain equivalent peptide cross-linkers as targets for MMPs (26). For the isolation of cells encapsulated in SAPHs, any non-specific proteolysis of SAPs would also degrade cell-surface proteins and, upon cell rupture, all cellular proteins. Instead, a method for protein analysis of in situ cells must focus on the complete solubilization of both cellular proteins and the SAPH, with a view to separate out the SAPs at a later stage. As such, the focus of this study is to identify a method for analyzing cellular proteins extracted from cells encapsulated in SAPHs. For this purpose, FEFKFEFK SAPHs were selected due to their popularity and ability to form a stable hydrogel suitable for cell encapsulation (27-29).

\section{Materials and methods}

\section{3-D cell encapsulation and} sample preparation

Human endothelial kidney cells (HEK293A, R70507; ThermoFisher Scientific, Loughborough, UK) were maintained under standard culture conditions in Dulbecco's modified Eagle's medium (DMEM) supplemented with $10 \%$ fetal bovine serum (FBS),

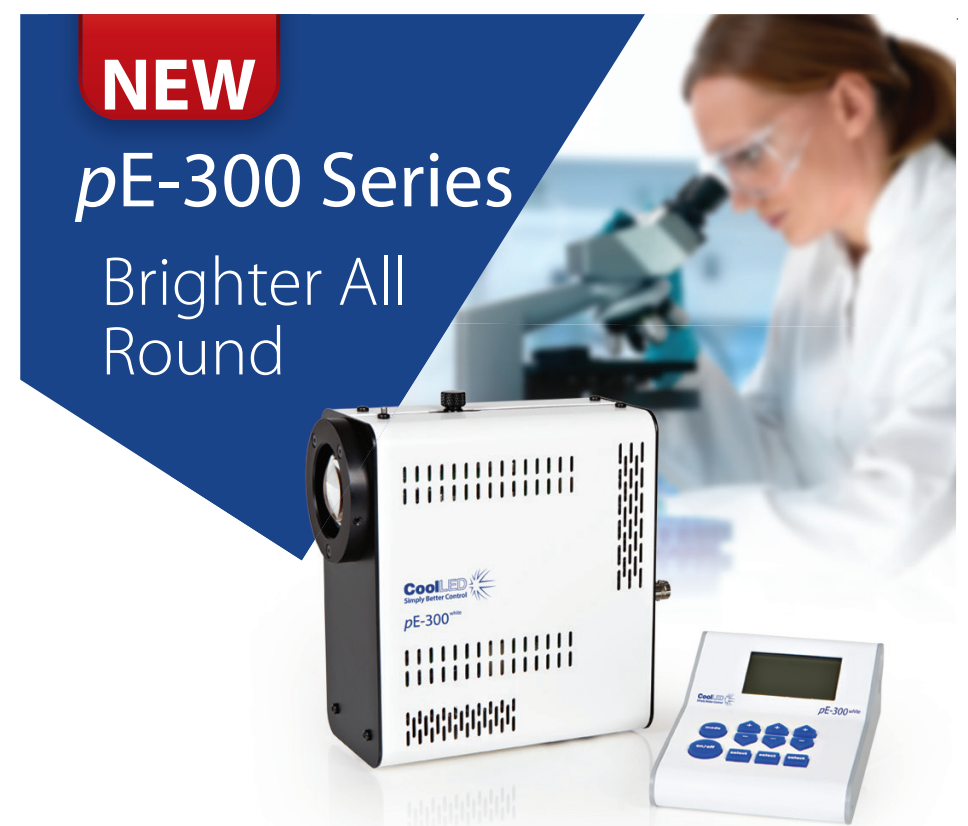

Double the intensity, half the power consumption, quadruple the efficiency

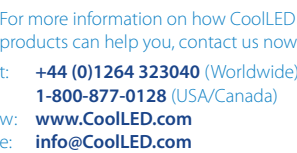

info@CoolLED.com
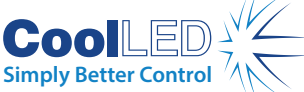

1\% penicillin/streptomycin, and MEM non-essential amino acids solution (1x). For serum-free conditions, cells were cultured as above, but upon cell-seeding, DMEM was prepared without the addition of FBS, and cells were washed with serum-free media twice to remove any residual serum.

Solutions of the oligopeptide FEFKFEFK (designated as F8) $(\mathrm{HCl}$ salt; $>95 \%$ purity) (BioMatik, Wilmington, DE) were prepared by dissolving the peptide to a concentration of $1 \%, 2 \%$, or $3 \%(\mathrm{w} / \mathrm{v})$ in filter-sterilized $\mathrm{ddH}_{2} \mathrm{O}$ water $\left(\mathrm{FSH}_{2} \mathrm{O}\right)$. The peptide solutions were titrated with $\mathrm{NaOH}$ until they started to form a weak gel ( $\mathrm{pH} 3.5-4)$. For cell encapsulation, $90 \mu \mathrm{l}$ gel was pipetted using a precision positive displacement microliter pipette (Microman; Gilson, Bedfordshire, UK) into 24-well cell culture inserts. Then, 10 $\mu$ l of either cell suspension $\left(2 \times 10^{7}\right.$ cells/ $\mathrm{mL}$ ) or media-only blank samples (- cell) was physically mixed into the gel (F8) or 90 ul Dulbecco's PBS (dPBS) (control). All samples were then incubated in standard culture conditions for 30 min, media was removed, and samples snap-frozen with liquid nitrogen. Samples were stored at $-80^{\circ} \mathrm{C}$ until processed.

Protein extraction

Hydrogel samples were thawed in 100 $\mu$ lysis buffer, either (i) radioimmunoprecipitation assay buffer (RIPA) buffer (1\% IGEPAL, $0.5 \%$ sodium deoxycholate, $0.1 \%-2 \%$ SDS (as specified); $0.5 \mathrm{mM}$ PMSF, $0.5 \mu \mathrm{g}$ leupeptin, $0.5 \mu \mathrm{g}$ aprotinin, $0.025 \mu \mathrm{g}$ pepstatin $\mathrm{A}, 1 \mathrm{mM}$ sodium orthovanadate, in dPBS) or (ii) urea buffer (8 M urea, $2 \mathrm{M}$ thiourea, $100 \mathrm{mM}$ Tris- $\mathrm{HCl}$, $5 \mathrm{mM}$ DTT, pH 8.0). The samples then underwent cycles of sonication (Covaris S220 ultrasonicator; Covaris, Brighton, UK), with each cycle of sonication lasting $180 \mathrm{~s}(40 \mathrm{~W})$. When using RIPA buffer, hydrogel samples underwent a total of three cycles of sonication; however, when using urea buffer, hydrogel samples were sonicated until complete dissolution was achieved (i.e., 2 cycles of sonication for $1 \%$ F8 and 3 cycles for 2\% and 3\% F8). Cell-only controls only underwent one cycle of sonication for either buffer used. Following each cycle of sonication, the mixture was centrifuged $(4000 \times g$ for 5 min at $4^{\circ} \mathrm{C}$ ), the supernatant was collected, and $100 \mu \mathrm{l}$ fresh buffer was added to the pellet before the sonication process was repeated. Total protein was then calcu- 
A

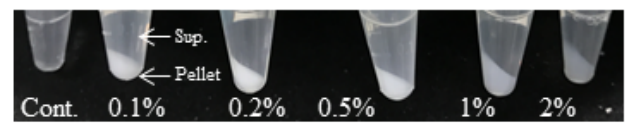

B

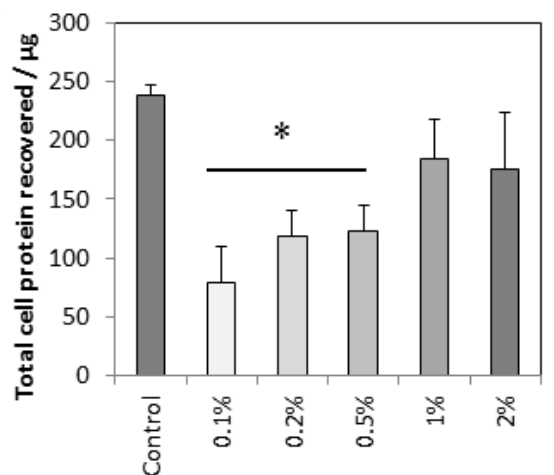

D

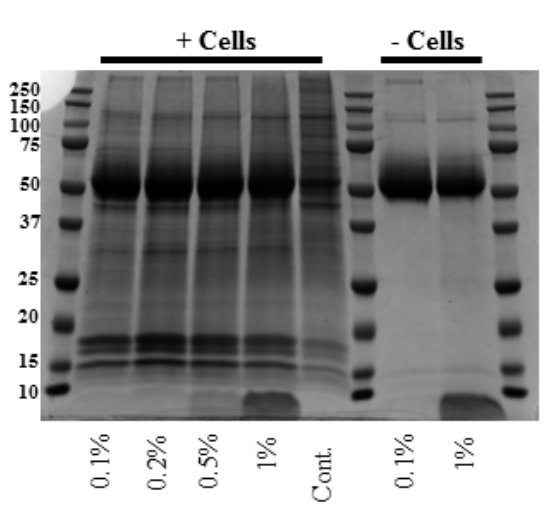

$\mathbf{F}$

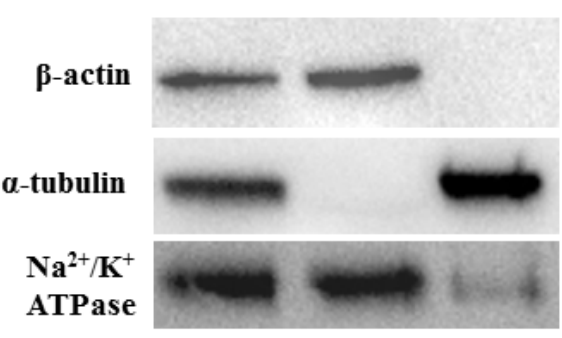

C

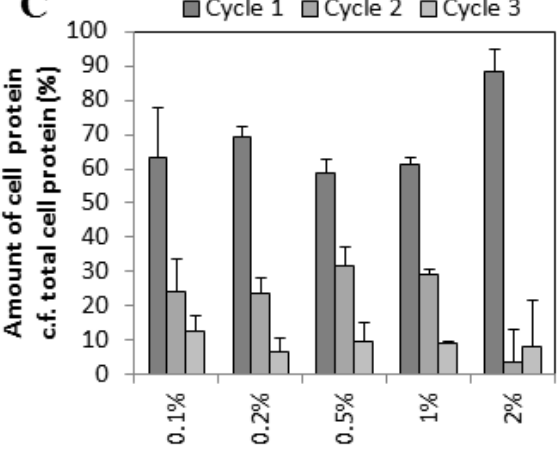

$\mathbf{E}$

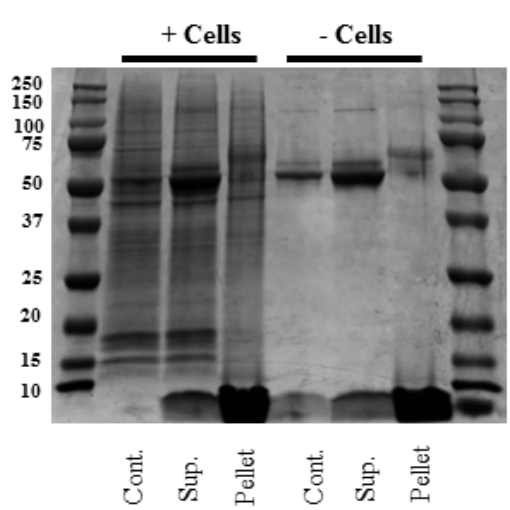

Figure 1. Protein extraction, SDS-PAGE and western blot analysis of HEK293 cells $\left(2 \times 10^{6}\right)$ encapsulated in $\mathrm{F} 8$ peptide hydrogel $(1 \% \mathrm{w} / \mathrm{v})$ when using detergent-based RIPA lysis buffer. Cells suspended in PBS were used as controls (Cont.), and blank samples (- cells) were prepared by adding equal volumes of medium in place of the cell suspension. (A) Images of cell-seeded self-assembling peptide hydrogel (SAPH) following three cycles of sonication with SDS RIPA buffer or one cycle for the control (cells in PBS). Figure labels depict SDS concentration in RIPA buffer. For the control, protein was extracted by 1 cycle of sonication using $0.1 \%$ SDS RIPA buffer. (B) Total cellular protein extracted ( $\mu \mathrm{g})$, following 3 cycles of sonication, was compared when varying the concentration of SDS from $0.1 \%$ to $2 \%$ in RIPA buffer. (C) For each concentration of SDS, the total cellular protein extracted is represented as a percentage of cellular protein extracted per cycle of sonication (1, 2 or 3$)$. For both graphs, the mean \pm SEM of three independent samples was calculated following background subtraction of the corresponding blank samples. Mean values were compared with the control using an unpaired $t$-test $(P \leq 0.05)$. (D,E) Coomassie Blue staining of protein extracts (20 $\mu \mathrm{g})$ separated by $12 \%$ SDS-PAGE. For blank samples (- cells), equal volumes of sample were loaded. (D) Protein separation profiles of cell lysates extracted from cells encapsulated in F8 hydrogels when varying the concentration of SDS in the extraction buffer. (E) Protein separation profiles of the supernatant (SUP.) and pellet of F8 hydrogel following extraction with $1 \%$ SDS RIPA buffer. The gel pellet was solubilized using a urea buffer ( $8 \mathrm{M}$ urea, $2 \mathrm{M}$ thiourea, $5 \mathrm{mM}$ DTT, $100 \mathrm{mM}$ Tris-Hcl, pH8.0). (F) Western blot analysis of cell lysates $(20 \mu \mathrm{g})$ to detect the presence of the endogenous proteins $\beta$-actin, $\alpha$-tubulin, and $\mathrm{Na}^{2+} / \mathrm{K}^{+}$ATPase.

lated based on sample volume (determined by measuring sample weight) and protein concentration. Total cellular protein was then calculated by subtracting the corresponding blank (- cell) sample. For samples lysed in RIPA, protein concentration was determined by measuring the absorbance in the 1600-1700 $\mathrm{nm}$ range (amide I bond) using infrared spectroscopy (Direct Detect Infrared Spectrometer; Millipore, Hertfordshire, UK). The total cellular protein for each supernatant was calculated separately and then added to determine the overall cellular protein recovered from the sample. For each sample, supernatants were combined before use in downstream applications. For samples lysed in urea buffer, the overall protein concentration was calculated using the Pierce 660 nm Protein Assay (ThermoFisher) and compared with a set of albumin standards (ThermoFisher).

For the gel pellet, F8 hydrogels were treated as above using RIPA (1\% SDS RIPA), but after 3 cycles of sonication the supernatant was replaced with $100 \mu \mathrm{l}$ urea buffer, and an additional sonication cycle was carried out. The concentration of protein in the gel pellet was estimated based on the difference between the amount of cellular protein recovered from the supernatants and the value obtained for the corresponding cell-only control. Images of the gel pellet were taken using a digital camera.

Gel staining and western blot analysis For RIPA samples only, total protein lysates were concentrated using filtration columns with a 3000 molecular weight cut-off (MWCO) (Millipore). Equal amounts of total cellular protein were separated by $12 \%$ SDS-PAGE (Bio-Rad, Hertfordshire, UK). For RIPA samples, the concentration of SDS was adjusted to $1 \%$, mixed with Laemmli buffer (ThermoFisher), and heated at $95^{\circ} \mathrm{C}$ for $5 \mathrm{~min}$. For urea buffer samples, the sample was mixed with Laemmli buffer without heating at high temperature. Gels were then either stained with Coomassie Blue G-250 (ThermoFisher) or transferred to a nitrocellulose membrane using a Trans-blot Turbo Transfer System (Bio-Rad) for western blot analyses. Membranes were blocked in 5\% milk before incubation with the following antibodies: anti- $\beta$ actin antibody

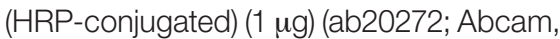
Cambridge, UK; RRID: AB_445482); anti- $\alpha$ 
tubulin antibody (HRP-conjugated) (1 $\mu \mathrm{g})$ (ab40742; Abcam; RRID: AB_880625); and anti-sodium potassium ATPase antibody (HRP-conjugated) (1 $\mu \mathrm{g}$ ) (ab185065; Abcam) in 1\% milk. Protein bands were detected with ECL western blotting detection reagents (GE Healthcare, Little Chalfont Bucks, UK). The Precision Plus Protein Dual Color Standards were used as molecular weight markers (Bio-Rad).

Statistical analysis

For comparison of protein concentrations, the mean \pm SEM was calculated from three independent samples. A two-sample $t$-test was performed, and statistically significance compared with the control was determined when $P \leq 0.05$.

\section{Results and discussion}

\section{Detergent-based solubilization}

The detergent SDS is widely used as an effective agent for membrane disruption and the solubilization of cellular proteins (2). SDS is an anionic surfactant that acts to both coat hydrophobic regions of proteins with a negative charge and mask positive charges in proteins. In theory, SDS should interact with both the hydrophilic and hydrophobic face of the $\beta$-sheet-forming FEFKFEFK (F8) peptide $(30,31)$, coating the F8 peptide with a negative charge that upon dissociation of the peptide fibers should hinder their ability to self-assemble (32). Therefore, attempts were made to solubilize the cellseeded F8 hydrogel through sonication with a detergent-based buffer (RIPA buffer; see above for details) in which the SDS content was varied from $0.1 \%$ to $2 \%$ with a view to accommodate the abundance of F8 peptide present (Figure 1). In brief, HEK293 cells were encapsulated in F8 hydrogel (1\% w/v), conditioned with media for $30 \mathrm{~min}$, and then snap-frozen in liquid nitrogen until processed. Cells were also suspended in PBS as controls. As expected, control samples were completely solubilized after sonication in $0.1 \%$ SDS-RIPA buffer. However, the F8 hydrogel formed a large pellet, which failed to solubilize even after three separate cycles of sonication, despite increasing the concentration of SDS (Figure 1A). After each cycle of sonication the supernatant was collected and replaced with $100 \mu \mathrm{l}$ fresh RIPA buffer. Despite incomplete solubilization of the F8 peptide, cellular proteins were still
$\mathbf{A}$

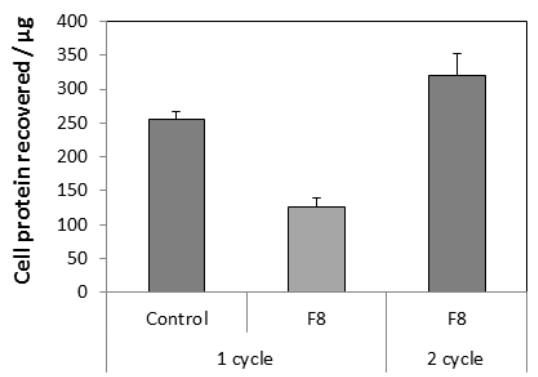

C

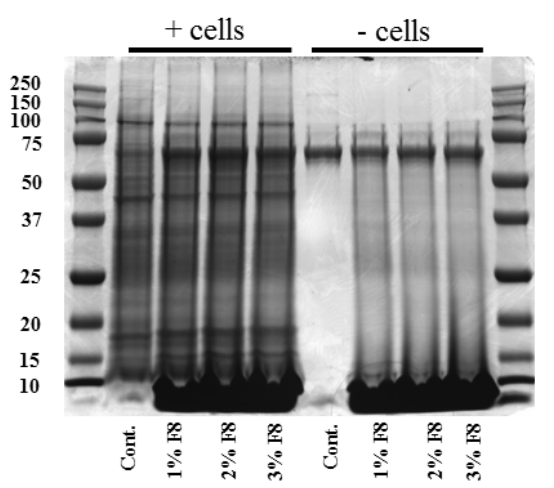

B

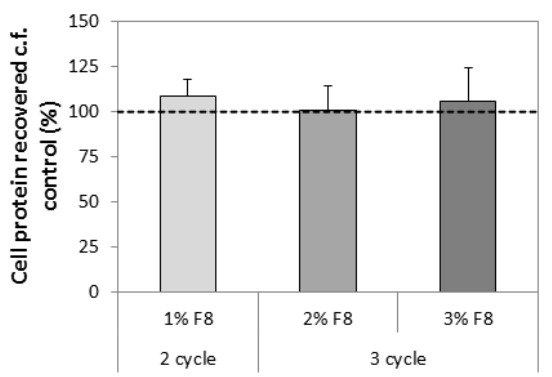

D

Figure 2. Protein extraction, SDS-PAGE, and western blot analysis of HEK293 cells $\left(2 \times 10^{6}\right)$ encapsulated in $\mathbf{F} 8$ peptide hydrogels $(1 \%, 2 \%$, and $3 \% \mathrm{w} / \mathrm{v})$ when using a urea/thiourea lysis buffer. Cells suspended in PBS were used as controls (Cont.), and blank samples (- cells) were prepared by adding equal volumes of medium in place of the cell suspension. (A) Total cellular protein recovered from cells encapsulated in 1\% F8 (w/v) hydrogels following either 1 or 2 cycles of sonication in urea/thiourea lysis buffer. (B) Total cellular protein recovered (percentage), compared with the control, from different concentrations of $\mathrm{F} 8$ hydrogels ( $1 \%, 2 \%$ and $3 \% \mathrm{w} / \mathrm{v}$ ) following either 2 or 3 cycles of sonication. The number of cycles of sonication corresponds to complete solubilization of the hydrogel. The dotted line represents the percentage of protein expected (100\%). (C) Coomassie Blue staining of protein extracts (15 $\mu \mathrm{g}$ ) separated by $12 \%$ SDS-PAGE. For blank samples (- cells), equal volumes of sample were loaded. D) Western blot analysis of cell lysates (15 $\mu \mathrm{g})$ extracted from F8 hydrogels (1\%, $2 \%$ and $3 \% \mathrm{w} / \mathrm{v}$ ) to detect the presence of the endogenous proteins $\beta$-actin and $\alpha$-tubulin.

detected in the supernatant, confirming that sonication in RIPA buffer indeed lysed encapsulated cells. Figure 1B shows the total amount of cellular protein extracted from all three cycles of sonication. By comparing total cellular protein recovered from cells encapsulated in F8 hydrogels using 0.1\% SDS-RIPA buffer $(79.8 \pm 29.6$ $\mu \mathrm{g}$ ) and the control (also 0.1\% SDS-RIPA buffer) $(237.7 \pm 8.8 \mu \mathrm{g})$, it can be seen that a significant amount of protein remained trapped in the gel pellet and is not recovered. When the concentration of SDS in RIPA buffer was increased, a gradual increase in the total amount of cellular protein recovered was observed. The maximum amount of cellular protein was recovered when using 1\% SDS-RIPA buffer (183.6 $\pm 77.3 \mu \mathrm{g} ; 77.3 \%$ of control). Figure $1 \mathrm{C}$ shows the percentage of protein obtained after each cycle of sonication, with respect to the total amount of cellular protein recovered for each composition of RIPA buffer tested. Despite differences in total cellular protein, the percentage of protein (compared to total cellular protein) recovered from each cycle of sonication was comparable between all five compositions of RIPA buffer. For all compositions of RIPA buffer, the percentage of cellular protein obtained from the first cycle of sonication was typically $\geq 60 \%$ and only $<10 \%$ for the final (third) cycle. As such, there would be little advantage to introducing additional cycles of sonication.

Cellular protein extracts were concentrated using spin column-based filtration (3000 MWCO), and the SDS concentration was adjusted to $1 \%$ before equivalent amounts of cellular protein $(20 \mu \mathrm{g})$ were separated on $12 \%$ polyacrylamide gels. The separation profiles following Coomassie Blue staining were comparable for cell lysates extracted across all five RIPA buffer compositions (Figure 1D), with the notable exception of a band $<10 \mathrm{kDa}$ that increased in intensity with increasing SDS concentration. This band 
was absent in the control and corresponds to the F8 peptide (molecular weight: $1121.28 \mathrm{~g} / \mathrm{mol}$ ) (Supplementary Figure S1), suggesting partial solubilization of the peptide by SDS. The presence of any F8 peptide carried over into the supernatant did not affect the separation of total cellular protein, at least for any of the SDS concentrations tested. The similarity in separation profiles across the different RIPA buffer compositions suggests no preferential retention of specific proteins; simply more protein appears to be homogeneously extracted from the gel pellet with increasing SDS concentration, which correlates with increasing F8 solubilization. However, when comparing the separation profiles against the cell-only control, there appears to be fewer distinct bands overall, with a complete absence of protein bands between $150 \mathrm{kDa}$ and $250 \mathrm{kDa}$. The bands present in the blank samples (- cells) represent serum proteins from cell culture media (Supplementary Figure S1), with the most intense band correlating with albumin ( 66.5 kDa).

To verify that cellular proteins remain trapped in the F8 hydrogel aggregate, gel pellets were dissolved following sonication with urea buffer (8 M urea, 2 $\mathrm{M}$ thiourea, $5 \mathrm{mM}$ DTT, $100 \mathrm{mM}$ Tris-HCl). Urea, a chaotropic salt, was investigated for its ability to disrupt hydrogen bonding (33) and reduce hydrophobic interactions (34), the two key interactions involved in peptide self-assembly into $\beta$-sheet rich fibers (35). In fact, urea has been noted to increase protein yield following protein precipitation (36) and together with thiourea was shown to significantly improve protein solubilization $(37,38)$. Moreover, it has been shown that urea is an effective agent for solubilizing poorly soluble (hydrophobic) membraneassociated proteins (39), proving more effective than RIPA buffer when tested with small heat-shock proteins (40). As expected, following solubilization of the gel pellet, the sample stained positive for an array of protein bands not seen in the blank (- cell) sample (Figure 1E). Interestingly, the separation profiles produced from the gel pellet and the supernatant differed, with some bands only being present in either the gel pellet or the supernatant.

To determine if there was retention of specific proteins in the gel pellet, western blot analysis of three different endogenous proteins was performed (Figure 1F). Equal amounts of cellular protein $(20 \mu \mathrm{g})$ from the supernatant and gel pellet were compared. The assumption was that the relative expression of each protein should be comparable if cellular protein is trapped homogeneously. However, western blot analysis revealed heterogeneity between the proteins detected in the supernatant and gel pellet. The expression of $\beta$-actin was only detected in the supernatant, whereas $\alpha$-tubulin was only detected in the gel pellet. The expression of $\mathrm{Na}^{2+} / \mathrm{K}^{+}$ ATPase was detected mainly in the supernatant, although some signal could be detected in the gel pellet. Moreover, we can confirm that the presence of serum proteins did not affect the ability to detect these three endogenous proteins (Supplementary Figure S1).

Overall, our data indicate that SDS on its own is unable to fully solubilize F8 and that complete solubilization of the F8 hydrogel is indeed required for western blot analysis to prevent loss of specific proteins to the gel pellet.

\section{Urea/thiourea-based solubilization}

Urea buffer was next tested for its ability to completely solubilize the F8 hydrogel without prior sonication in RIPA buffer. In contrast with RIPA buffer, multiple cycles of sonication in urea buffer resulted in the complete solubilization of the hydrogel construct. In fact, after 2 cycles of sonication, the F8 hydrogel $(1 \% \mathrm{w} / \mathrm{v})$ was completely solubilized, enabling the total recovery of cellular proteins (sample: $319.8 \pm 32.2 \mu \mathrm{g}$; control: $255 \pm 12.3 \mu \mathrm{g}$ ) (Figure 2A). However, incomplete solubilization of the F8 hydrogel after just 1 cycle of sonication resulted in the recovery of only $52.8 \pm 8 \%$ of the total cellular protein compared with the control, emphasizing once again the importance of fully solubilizing the SAPH matrix.

As mentioned earlier, the properties of SAPHs can be tailored by altering the peptide concentration. Therefore, this method of solubilization in urea buffer was also tested with cells encapsulated in F8 hydrogels of higher peptide concentrations (2\% and $3 \% \mathrm{w} / \mathrm{v}$ ) (Figure 2, B-D). Following complete solubilization, the amount of cellular protein recovered was comparable to the control for all three concentrations of F8 hydrogel (Figure $2 \mathrm{~B})$. The percentage of cellular protein recovered, compared with the control, was $108.9 \pm 9 \%, 101 \pm 13.2 \%$, and 105.5 $\pm 19.1 \%$ for $1 \%, 2 \%$, and $3 \% \mathrm{w} / \mathrm{v}$ F8, respectively. Importantly, the 2 hydrogels with a higher peptide concentration (2\% and $3 \% \mathrm{w} / \mathrm{v}$ ) required an additional cycle (for 3 cycles total) of sonication to achieve complete solubilization. For all 3 concentrations of F8 hydrogel, an equal amount of cellular protein $(15 \mu \mathrm{g})$ was separated on a $12 \%$ polyacrylamide gel (Figure 2C). The protein separation profiles for all three concentrations of F8 hydrogel were comparable to the control. Interestingly, even at the highest concentration (3\%), the peptide did not interfere with cellular protein separation. Instead, the F8 peptide also separated out on the polyacrylamide gel and is represented by a dominant band $<10 \mathrm{kDa}$, as seen when using RIPA buffer. However, for all concentrations the peptide forms a smear that begins at $\sim 70 \mathrm{kDa}$ and extends to the dominant band at $<10$ $\mathrm{kDa}$, most obviously within the blank samples (- cells). One explanation could be that as the urea is diluted out of the sample during electrophoresis, the peptide begins to self-assemble, forming peptide complexes of various sizes. Nevertheless, western blot analysis revealed clear distinct bands for both $\beta$-actin and $\alpha$-tubulin for all concentrations of F8 hydrogel, with $\alpha$-tubulin having been trapped in the gel pellet previously (Figure 2D). This demonstrates that even at the highest concentration of F8, the peptide is not interfering with protein size separation or antibody detection.

Western blot analysis of cells encapsulated in F8 peptide hydrogels requires complete solubilization of the hydrogel construct. Complete solubilization of both F8 peptide and cellular protein was achieved using multiple cycles of sonication with urea buffer but not when using RIPA buffer. The latter caused the F8 hydrogel to aggregate and trap cellular proteins, while the former had the ability to break hydrogen bonding, which is thought to be key to solubilizing $\mathrm{SAPH}$. Therefore, this method should also apply to all SAPHs composed of $\beta$-sheet-forming peptides such as RADA, KLD, and Q11, mentioned above. Following solubilization in urea buffer, the F8 peptide did not interfere with western blot analysis of endogenous cellular proteins. 


\section{Author contributions}

K.A.B. conceived, designed, and performed all of the experiments and related data analysis. K.A.B. wrote the paper in collaboration with A.S., A.F.M., and D.O. The study was supervised by A.S., who provided advice and guidance.

\section{Acknowledgments}

The authors would like to thank Ronan O'Cualain (BMSCF) and Andrew Smith (MIB, Manchester, UK) for useful experimental advice. The authors acknowledge the MRC/EPSRC for funding K.A.B.'s PhD scholarship through the Manchester's Centre of Doctoral Training (CDT) in Regenerative Medicine and the EPSRC for providing additional funding to this research through A.S.'s Fellowship grant (EP/K016210/1). All research data supporting this work are directly available within this publication.

\section{Competing interests}

The authors declare no competing interests.

\section{References}

1. Towbin, H., T. Staehelin, and J. Gordon. 1979. Electrophoretic transfer of proteins from polyacrylamide gels to nitrocellulose sheets - procedure and some applications. Proc. Natl. Acad. Sci. USA 76:4350-4354

2. Bass, J.J., D.J. Wilkinson, D. Rankin, B.E. Phillips, N.J. Szewczyk, K. Smith, and P.J. Atherton. 2017. An overview of technical considerations for western blotting applications to physiological research. Scand. J. Med. Sci. Sports 27:4-25.

3. Jensen, E.C. 2012. The basics of western blotting. Anat. Rec. (Hoboken) 295:369-371.

4. Janes, K.A. 2015. An analysis of critical factors for quantitative immunoblotting. Sci. Signal. 8:rs2.

5. Shen, K., J. Sun, X.X. Cao, D.B. Zhou, and J. Li. 2015. Comparison of different buffers for protein extraction from formalin-fixed and paraffinembedded tissue specimens. PLoS One 10: e0142650.

6. Shevchenko, G., S. Musunuri, M. Wetterhall, and J. Bergquist. 2012. Comparison of extraction methods for the comprehensive analysis of mouse brain proteome using shotgun-based mass spectrometry. J. Proteome Res. 11:2441-2451.

7. Glatter, T., E. Ahrne, and A. Schmidt. 2015. Comparison of different sample preparation protocols reveals lysis buffer-specific extraction biases in gram-negative bacteria and human cells. J. Proteome Res. 14:4472-4485.

8. Baghirova, S., B.G. Hughes, M.J. Hendzel, and R. Schulz. 2015. Sequential fractionation and isolation of subcellular proteins from tissue or cultured cells. MethodsX 2:440-445.

9. Kondiah, P.J., Y.E. Choonara, P.P.D. Kondiah, T. Marimuthu, P. Kumar, L.C. du Toit, and V. Pillay. 2016. A review of injectable polymeric hydrogel systems for application in bone tissue engineering. Molecules 21:pii E1580.

10. Du, E.Y., A.D. Martin, C. Heu, and P. Thordarson. 2016. The use of hydrogels as biomimetic materials for 3d cell cultures. Aust J Chem. 70:1-8.

11. Slaughter, B.V., S.S. Khurshid, O.Z. Fisher, A. Khademhosseini, and N.A. Peppas. 2009. Hydrogels in regenerative medicine. Adv. Mater. 21:3307-3329.

12. Zhang, S., T. Holmes, C. Lockshin, and A. Rich. 1993. Spontaneous assembly of a self-complementary oligopeptide to form a stable macroscopic membrane. Proc. Natl. Acad. Sci. USA 90:33343338.

13. Zhang, S., C. Lockshin, R. Cook, and A. Rich. 1994. Unusually stable beta-sheet formation in an ionic self-complementary oligopeptide. Biopolymers 34:663-672

14. Cormier, A.R., X.D. Pang, M.I. Zimmerman, H.X. Zhou, and A.K. Paravastu. 2013. Molecular structure of rada16-i designer self-assembling peptide nanofibers. ACS Nano 7:7562-7572.

15. Kisiday, J., M. Jin, B. Kurz, H. Hung, C. Semino, S. Zhang, and A.J. Grodzinsky. 2002. Self-assembling peptide hydrogel fosters chondrocyte extracellular matrix production and cell division: Implications for cartilage tissue repair. Proc. Natl. Acad. Sci. USA 99:9996-10001.

16. Caplan, M.R., E.M. Schwartzfarb, S.G. Zhang, R.D. Kamm, and D.A. Lauffenburger. 2002 Control of self-assembling oligopeptide matrix formation through systematic variation of amino acid sequence. Biomaterials 23:219-227.

17. Hwang, W.M., D.M. Marini, R.D. Kamm, and S.Q. Zhang. 2003. Supramolecular structure of helical ribbons self-assembled from a $\beta$-sheet peptide. J. Chem. Phys. 118:389-397.

18. Jung, J.P., A.K. Nagaraj, E.K. Fox, J.S. Rudra, J.M. Devgun, and J.H. Collier. 2009. Co-assembling peptides as defined matrices for endothelial cells. Biomaterials 30:2400-2410,

19. Saiani, A., A. Mohammed, H. Frielinghaus, R. Collins, N. Hodson, C.M. Kielty, M.J. Sherratt, and A.F. Miller. 2009. Self-assembly and gelation properties of alpha-helix versus beta-sheet forming peptides. Soft Matter 5:193-202.

20. Mujeeb, A., A.F. Miller, A. Saiani, and J.E. Gough 2013. Self-assembled octapeptide scaffolds for in vitro chondrocyte culture. Acta Biomater. 9:46094617.

21. Diaz, L.A.C., J. Gough, A. Saiani, and A. Miller 2014. Human osteoblasts within soft peptide hydrogels promote mineralisation in vitro. J Tissue Eng. 5:1-12.

22. Sieminski, A.L., C.E. Semino, H. Gong, and R.D. Kamm. 2008. Primary sequence of ionic self-assembling peptide gels affects endothelial cell adhesion and capillary morphogenesis. J. Biomed. Mater. Res. A 87:494-504.

23. Huang, F., Q. Shen, and J.T. Zhao. 2013. Growth and differentiation of neural stem cells in a threedimensional collagen gel scaffold. Neural Regen. Res. 8:313-319.

24. Carrion, B., I.A. Janson, Y.P. Kong, and A.J. Putnam. 2014. A safe and efficient method to retrieve mesenchymal stem cells from threedimensional fibrin gels. Tissue Eng. Part C Methods 20:252-263.

25. Burdick, J.A. and G.D. Prestwich. 2011. Hyaluronic acid hydrogels for biomedical applications. Adv. Mater. 23:H41-H56.

26. Lutolf, M.P., J.L. Lauer-Fields, H.G. Schmoekel, A.T. Metters, F.E. Weber, G.B. Fields, and J.A.
Hubbell. 2003. Synthetic matrix metalloproteinasesensitive hydrogels for the conduction of tissue regeneration: Engineering cell-invasion characteristics. Proc. Natl. Acad. Sci. USA 100:5413-5418.

27. Marini, D.M., W. Hwang, D.A. Lauffenburger, S.G. Zhang, and R.D. Kamm. 2002. Left-handed helical ribbon intermediates in the self-assembly of a $\beta$-sheet peptide. Nano Lett. 2:295-299.

28. Zhang, S. 2003. Building from the bottom up. Mater. Today 6:20-27.

29. Rudra, J.S., T. Sun, K.C. Bird, M.D. Daniels, J.Z Gasiorowski, A.S. Chong, and J.H. Collier. 2012. Modulating adaptive immune responses to peptide self-assemblies. ACS Nano 6:1557-1564.

30. Elsawy, M.A., A.M. Smith, N. Hodson, A. Squires, A.F. Miller, and A. Saiani. 2016. Modification of $\beta$-sheet forming peptide hydrophobic face: effect on self-assembly and gelation. Langmuir 32:4917-4923.

31. Gao, J., C. Tang, M.A. Elsawy, A.M. Smith, A.F. Miller, and A. Saiani. 2017. Controlling self-assembling peptide hydrogel properties through network topology. Biomacromolecules 18:826-834.

32. Yokoi, H., T. Kinoshita, and S.G. Zhang. 2005 Dynamic reassembly of peptide RADA16 nanofiber scaffold. Proc. Natl. Acad. Sci. USA 102:8414-8419.

33. Lim, W.K., J. Rösgen, and S.W. Englander. 2009 Urea, but not guanidinium, destabilizes proteins by forming hydrogen bonds to the peptide group. Proc. Natl. Acad. Sci. USA 106:2595-2600.

34. Zangi, R., R.H. Zhou, and B.J. Berne. 2009 Urea's action on hydrophobic interactions. J. Am. Chem. Soc. 131:1535-1541.

35. Caplan, M.R., P.N. Moore, S.G. Zhang, R.D. Kamm, and D.A. Lauffenburger. 2000. Selfassembly of a beta-sheet protein governed by relief of electrostatic repulsion relative to van der Waals attraction. Biomacromolecules 1:627-631.

36. Simões, A.E.S., D.M. Pereira, J.D. Amaral, A.F. Nunes, S.E. Gomes, P.M. Rodrigues, A.C. Lo, R. D'Hooge, et al. 2013. Efficient recovery of proteins from multiple source samples after trizol (R) or trizol (R) LS RNA extraction and long-term storage. BMC Genomics 14:181.

37. Rabilloud, T. 1998. Use of thiourea to increase the solubility of membrane proteins in two-dimensional electrophoresis. Electrophoresis 19:758-760.

38. Rabilloud, T., C. Adessi, A. Giraudel, and J. Lunardi. 1997. Improvement of the solubilization of proteins in two-dimensional electrophoresis with immobilized pH gradients. Electrophoresis 18:307316.

39. Cordwell, S.J. 2008. Sequential extraction of proteins by chemical reagents, p. 139-146. In A. Posch (Ed.), 2D PAGE: sample preparation and fractionation. Humana Press, Totowa, NJ.

40. Peach, M., N. Marsh, E.I. Miskiewicz, and D.J. MacPhee. 2015. Solubilization of proteins: the importance of lysis buffer choice, p. 49-60. In B.T. Kurien, and R.H. Scofield (Eds.), western blotting: methods and protocols. Humana Press Inc, Totowa, NJ.

Received 08 September 2017; accepted 19 October 2017.

Address correspondence to Alberto Saiani, School of Materials, The University of Manchester, Oxford Road, M13 9PL Manchester, UK. E-mail: a.saiani@ manchester.ac.uk

To purchase reprints of this article, contact: biotechniques@fosterprinting.com 